\title{
Extended reciprocal space observation of artificial spin ice with x-ray resonant magnetic scattering
}

\author{
J. Perron,,${ }^{1,2,3,4}$ L. Anghinolfi,,${ }^{3,4,}$ B. Tudu,,${ }^{1,2, \dagger}$ N. Jaouen, ${ }^{5}$ J.-M. Tonnerre,${ }^{6,7}$ M. Sacchi, ${ }^{5,8,9}$ F. Nolting, ${ }^{4}$ J. Lüning, ${ }^{1,2}$ and \\ L. J. Heyderman ${ }^{3,4}$ \\ ${ }^{1}$ Sorbonne Universités, UPMC Univ Paris 06, UMR 7614, LCPMR, 75005 Paris, France \\ ${ }^{2}$ CNRS, UMR 7614, LCPMR, 75005 Paris, France \\ ${ }^{3}$ Laboratory for Mesoscopic Systems, Department of Materials, ETH Zürich, 8093 Zürich, Switzerland \\ ${ }^{4}$ Paul Scherrer Institut, 5232 Villigen PSI, Switzerland \\ ${ }^{5}$ Synchrotron SOLEIL, 91192 Gif-sur-Yvette, France \\ ${ }^{6}$ Université Grenoble Alpes, Institut Néel, 38042 Grenoble, France \\ ${ }^{7}$ CNRS, Institut Néel, 38042 Grenoble, France \\ ${ }^{8}$ Sorbonne Universités, UPMC Univ Paris 06, UMR 7588, INSP, 75005 Paris, France \\ ${ }^{9}$ CNRS, UMR 7588, INSP, 75005 Paris, France
}

(Received 23 October 2013; published 23 December 2013)

\begin{abstract}
Soft x-ray resonant magnetic scattering is an element-sensitive technique that enables the characterization of the magnetic properties of a wide variety of systems. Here we apply this technique to study lithographically produced artificial spin ice, a particular class of magnetically frustrated systems comprising arrays of nanomagnets. Using a CCD detector we can access a large fraction of the reciprocal space at once, allowing us to easily distinguish the signatures of the magnetic ground-state ordering. Comparing the dichroic signal at the position of the Bragg peaks with model calculations based on the kinematical theory of x-ray diffraction, we are able to determine the number of reversed moments as a function of applied magnetic field for each of the two sublattices. This study demonstrates the benefit of having direct access to a significant fraction of the reciprocal space, and opens the way towards more sophisticated x-ray based experiments on artificial spin ice such as scattering of coherent x-ray beams to explore the dynamics of thermally activated systems.
\end{abstract}

DOI: 10.1103/PhysRevB.88.214424

PACS number(s): 78.70.Ck, 75.25.-j, 75.75.-c

\section{INTRODUCTION}

Artificial spin ice is a class of metamaterials produced by electron beam lithography, ${ }^{1,2}$ which consists of dipolarcoupled nanomagnets placed at the sites of a square or kagome lattice. The configuration of the magnetic moments mimics the spin arrangements of the magnetically frustrated rare-earth pyrochlore paramagnets (spin ice). ${ }^{3}$ In the past decade artificial spin ice has attracted an increasing amount of interest due to a wide range of fascinating behavior arising from the geometrical frustration. ${ }^{1,4-9}$ Experimentally, these have been investigated primarily using real-space microscopy techniques. For example, magnetic force microscopy and Lorentz microscopy have been employed in pioneering studies to observe the magnetically frustrated nature of artificial spin ice ind $^{1,4}$ and later the ground-state ordering in as-grown samples. ${ }^{5,6}$ The presence of magnetic charges, referred to as emergent magnetic monopoles, and their propagation have been observed in artificial kagome ice using photoelectron emission spectroscopy ${ }^{7}$ and in artificial square ice using Lorentz microscopy. ${ }^{8}$ More recently, the nature of their motion has been clarified by Zeissler et al. in a kagome ice using scanning transmission x-ray microscopy. ${ }^{9}$

So far, ordering phenomena in artificial spin ice has been investigated mostly on systems exhibiting static moments at room temperature. Current research is moving, however, towards thermally activated systems, ${ }^{10-12}$ which can be realized by reducing the size of the nanomagnets. Experimentally, this poses a problem, since the required sizes approach the limit of the spatial resolution achievable with real-space microscopy techniques that provide simultaneously the required time resolution. It is in the context of these experimental limitations that scattering techniques become of interest in order to further push towards a higher combined spatial and temporal resolution. It is this promise to follow dynamics in artificial spin ice at faster time scales which is expected to compensate for the less intuitive interpretation of the obtained experimental data. We note that two-dimensional arrays of nanomagnets with the nanomagnet dimensions of tens to hundreds of nanometers are particularly well suited for x-ray scattering experiments in terms of intensity and surface sensitivities. This is particularly true for the soft x-ray energy range, which covers the magnetically dichroic strong $L_{2,3}$ absorption edges of the transition metals. Indeed, following the discovery of resonant magnetic $\mathrm{X}$-ray scattering ${ }^{13-16}$ and $\mathrm{X}$-ray magnetic circular dichroism (XMCD), ${ }^{17-19}$ soft x-ray resonant magnetic scattering (SXRMS) has proven to be a powerful technique for the detailed investigation of a wide variety of magnetic materials. $^{20-23}$

Morgan et al. $^{24}$ have performed a SXRMS study on an artificial spin ice. Using a photodiode they recorded the intensity of the specular reflection and off-specular Bragg peaks of an artificial square ice while stepping through a hysteresis loop. From the data obtained they could gain insight into the magnetization reversal processes of the two interacting magnetic sublattices. In the present work, we have also applied SXRMS to investigate the magnetic behavior of an artificial square ice but, in order to obtain a more detailed insight into the field dependence of different Bragg peaks, we used a two-dimensional CCD detector. This allowed us to unambiguously identify Bragg peaks arising uniquely from the magnetic long range order in the as-grown state of the artificial square ice. To interpret the experimental data, we have developed a simple code which is based on the 
kinematical theory of x-ray diffraction. By comparing the simulated scattering patterns to the observed field dependence of the magnetic contribution to the first order Bragg peaks, we are able to quantify the fraction of nanomagnets with reversed magnetization for each of the two sublattices.

\section{EXPERIMENTAL METHODS}

\section{A. Sample preparation}

The artificial square ice samples were fabricated using electron-beam lithography. ${ }^{7}$ A $70 \mathrm{~nm}$ thick polymethylmethacrylate (PMMA) layer is spin coated on a $\mathrm{Si}(100)$ substrate and patterned using an electron beam writer. After development a $20 \mathrm{~nm}$ thick Permalloy $\left(\mathrm{Ni}_{80} \mathrm{Fe}_{20}\right)$ layer is deposited by thermal evaporation and capped with a $5 \mathrm{~nm}$ thick aluminium layer to prevent oxidation. Unwanted metal and resist are then removed by lift-off. The nanomagnet length and width are $230 \mathrm{~nm}$ and $80 \mathrm{~nm}$, respectively, and the lattice parameter is $a=310 \mathrm{~nm}$. The scanning electron microscopy image in Fig. 1(a) shows a representative region of the $2 \times 2 \mathrm{~mm}^{2}$ array.

\section{B. Soft x-ray magnetic scattering}

The SXRMS measurements were performed at the SEXTANTS beamline of Synchrotron SOLEIL using the RESOXS diffractometer, ${ }^{25,26}$ which is equipped with two pairs of electromagnets to apply magnetic fields of up to $0.2 \mathrm{~T}$ in arbitrary directions parallel to the sample surface. To obtain high sensitivity to the in-plane magnetization of the nanomagnet array, a reflection geometry with an incident angle of $\theta=8^{\circ}$ was used [Fig. 1(b)]. The diffraction patterns were acquired with a Princeton charge-coupled device (CCD) camera. In this geometry the CCD detector's $2048 \times 2048$ pixels, each with a size of $13.5 \times 13.5 \mu \mathrm{m}^{2}$, cover a momentum transfer range of about $\Delta q_{x}= \pm 2 \times 10^{-1} \mathrm{~nm}^{-1}$ and $\Delta q_{z}= \pm 1 \mathrm{~nm}^{-1}$. Alignment of the CCD detector was performed in two steps. First, the position of the specular reflection was determined using a photodiode, and then the center of the CCD detector was placed at that position with a beam stop protecting the detector against damage from the intense specular beam. While pure charge scattering is obtained for off-resonant photon energies, additional magnetic scattering contrast is observed by tuning the photon energy to the magnetically dichroic $\mathrm{Fe}$ $L_{3}$ absorption edge $(706.8 \mathrm{eV})$. The dichroic contrast of a Bragg peak can then be extracted by calculating the difference between the intensities recorded with right $\left(I_{\text {right }}\right)$ and left $\left(I_{\text {left }}\right)$ circularly polarized $\mathrm{x}$ rays:

$$
I_{\text {dic }}=I_{\text {right }}-I_{\text {left }} .
$$

For each polarization state, the intensity of the scattered beam, in the elastic regime, is related to the magnitude squared of the total atomic scattering factor: ${ }^{27}$

$$
I(\mathbf{q}) \propto\left|\sum_{j} f_{j}(\mathbf{q}, E) \exp \left(i \mathbf{q} \cdot \mathbf{r}_{j}\right)\right|^{2},
$$

where $f_{j}(\mathbf{q}, E)$ is the scattering factor of an atom located at position $\mathbf{r}_{j}, E$ the incident photon energy, and $\mathbf{q}$ is the momentum transfer defined as $\mathbf{q}=\mathbf{k}^{\prime}-\mathbf{k}$, with $\mathbf{k}$ and $\mathbf{k}^{\prime}$ the wave vector of

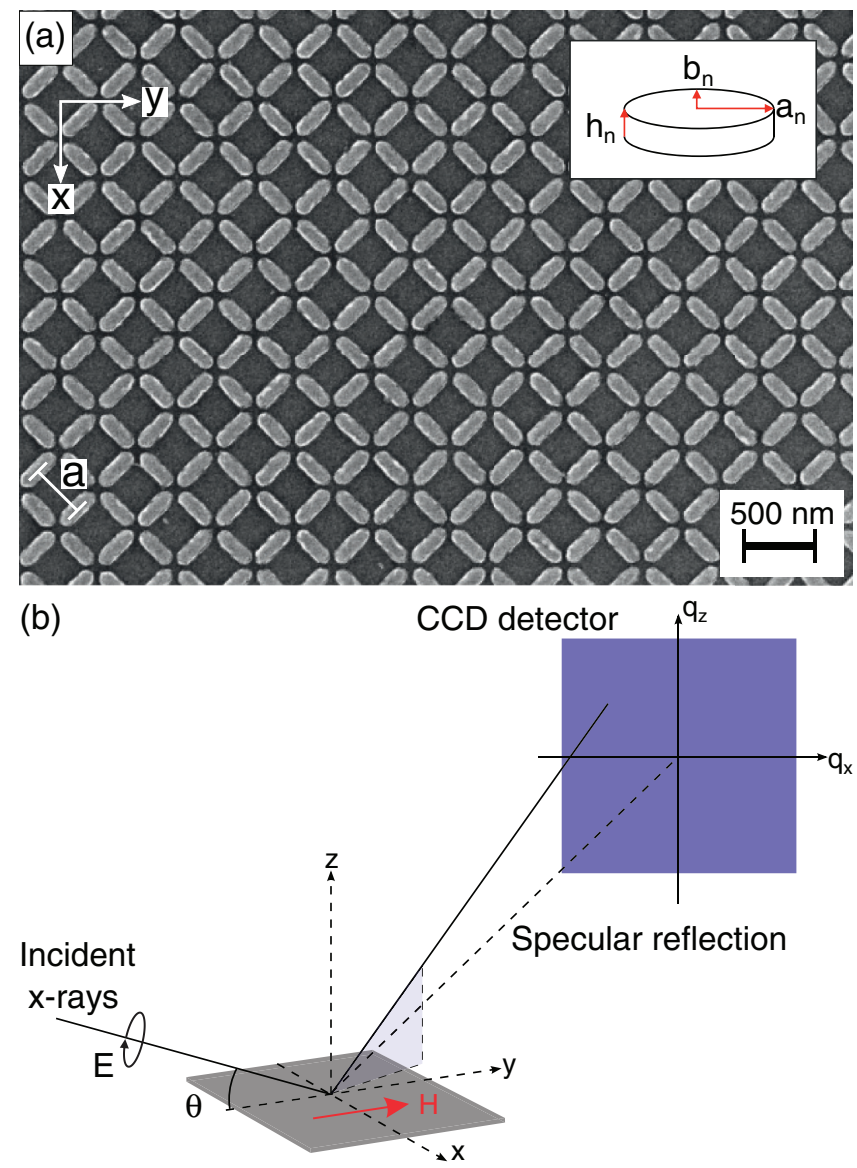

FIG. 1. (Color online) (a) SEM picture of part of the $2 \times 2 \mathrm{~mm}^{2}$ array of artificial square ice built from Permalloy nanomagnets of $230 \mathrm{~nm} \times 80 \mathrm{~nm} \times 20 \mathrm{~nm}$ (length $\times$ width $\times$ thickness). Inset: Illustration of the cylindrical approximation used to analytically represent the individual nanomagnets. The cylinders have height $h$ and elliptical cross section with semiaxes $a$ and $b$. (b) Experimental scattering setup. The sample surface is parallel to the $x-y$ plane, with the direction of the incident $\mathrm{x}$-ray beam and applied magnetic field, $H$, along $y$. The angle of incidence is $\theta=8^{\circ}$.

the incident and scattered beam, respectively. In the following analysis the $\mathbf{q}$ dependence of the atomic scattering factor can be neglected, since only small momentum transfer values can be reached in the soft $\mathrm{x}$-ray photon energy range.

For off-resonant photon energies, the atomic scattering factor is given by the Thomson scattering factor $f_{0}$, which takes the value of the total number of electrons $(Z)$ in the limit of negligible momentum transfer. It provides only structural information with no magnetic contribution. For photon energies close to an absorption edge, resonant scattering processes become relevant, and to a first approximation $f_{j}(E)$ can be expressed as ${ }^{15,16}$

$$
f_{j}(E) \approx\left[Z+f_{c}(E)\right]\left(\hat{\varepsilon}^{\prime *} \cdot \hat{\varepsilon}\right)-i f_{m}(E)\left(\hat{\varepsilon}^{*} \times \hat{\varepsilon}\right) \cdot \hat{m}_{j},
$$

where $\hat{\varepsilon}$ and $\hat{\varepsilon}^{\prime}$ are the polarization vectors of the incident and scattered photons, respectively, and $\hat{m}_{j}$ is the unit vector of the magnetic moment at the $j$ th site. The first resonant term $\left[f_{c}(E)\right]$ is the resonant modification of the charge scattering term, while the second term $\left[f_{m}(E)\right]$ depends linearly on 
the magnetization vector and yields the sensitivity to the local magnetization. Due to the linear dependence on the magnetization vector, the simplest way to extract the magnetic contribution to the recorded scattering pattern is to determine the difference in intensity for left and right circular polarization of the incoming beam $\left(I_{\text {right }}-I_{\text {left }}\right)$.

In the following, we will refer to Bragg peaks with $h^{2}+l^{2}=1$ as first order Bragg peaks and with $h^{2}+l^{2}=2$ as second order Bragg peaks, with $h$ and $l$ being their Miller indices in reciprocal space.

\section{RESULTS AND DISCUSSION}

\section{A. Ordering in as-grown artificial square ice}

SXRMS patterns recorded on an artificial square ice in the as-grown state are shown in Fig. 2. As illustrated in Fig. 2(a), the nanomagnet array is oriented such that the incident beam is parallel to the diagonal of the square lattice, which yields equal sensitivity to the magnetization of both sets of nanomagnets indicated in white and gray (apart from a slight misalignment; see below). Note that this was not the case in the study of Morgan et al. in Ref. 24, who chose to have one of the sets of nanomagnets oriented parallel to the incident beam.

The pattern recorded for an off-resonant photon energy $(690 \mathrm{eV})$ is shown in Fig. 2(b), which results from pure charge scattering of the nanomagnets, since there is no magnetic contribution to the nonresonant atomic form factors. In order to understand the position of the Bragg peaks, one needs to consider the structure of the artificial square ice illustrated in Fig. 2(a). The unit cell contains two sets of nanomagnets with different orientations (indicated in white and gray) and the array is reproduced with the help of the two indicated lattice vectors of equal length $a$. Correspondingly, the Bragg peaks form a square lattice with periodicity $2 \pi / a$. The first order peaks of this lattice are connected by the dashed lines in Fig. 2(b). Note that these lines do not form a square, but rather resemble a compressed parallelogram due to the distortion intrinsic to the reflection geometry. Furthermore, the Bragg peaks are situated on arcs, which is a consequence of the projection of the Ewald sphere on the flat detector plane. Finally, we note that the arcs are slightly tilted due to an imperfect alignment of sample and CCD detector with respect to the incident and scattered beam.

By tuning the photon energy to the $\mathrm{Fe} L_{3}$ edge $(706.8 \mathrm{eV})$, the scattering process also becomes sensitive to the magnetization of the nanomagnets and we obtain the SXRMS pattern shown in Fig. 2(d). Comparing this with the pattern in Fig. 2(b), one finds that additional Bragg peaks are present at resonance (highlighted in red). These additional Bragg peaks are of pure magnetic origin and are located in-between the positions of the already observed nonresonant Bragg peaks. The magnetic Bragg peaks are a signature of long range ground-state ordering of the moments of the nanomagnets as previously observed in comparable as-grown samples. ${ }^{5,6}$ As illustrated in Fig. 2(c), this ground state consists of an antiferromagnetic ordering of the magnetic moments, which forms alternating rows of nanomagnets with opposite magnetization directions. The unit cell of this magnetic ground state, indicated in Fig. 2(c), contains four nanomagnets (while there are two in the structural unit cell). The orthogonal lattice vectors are of

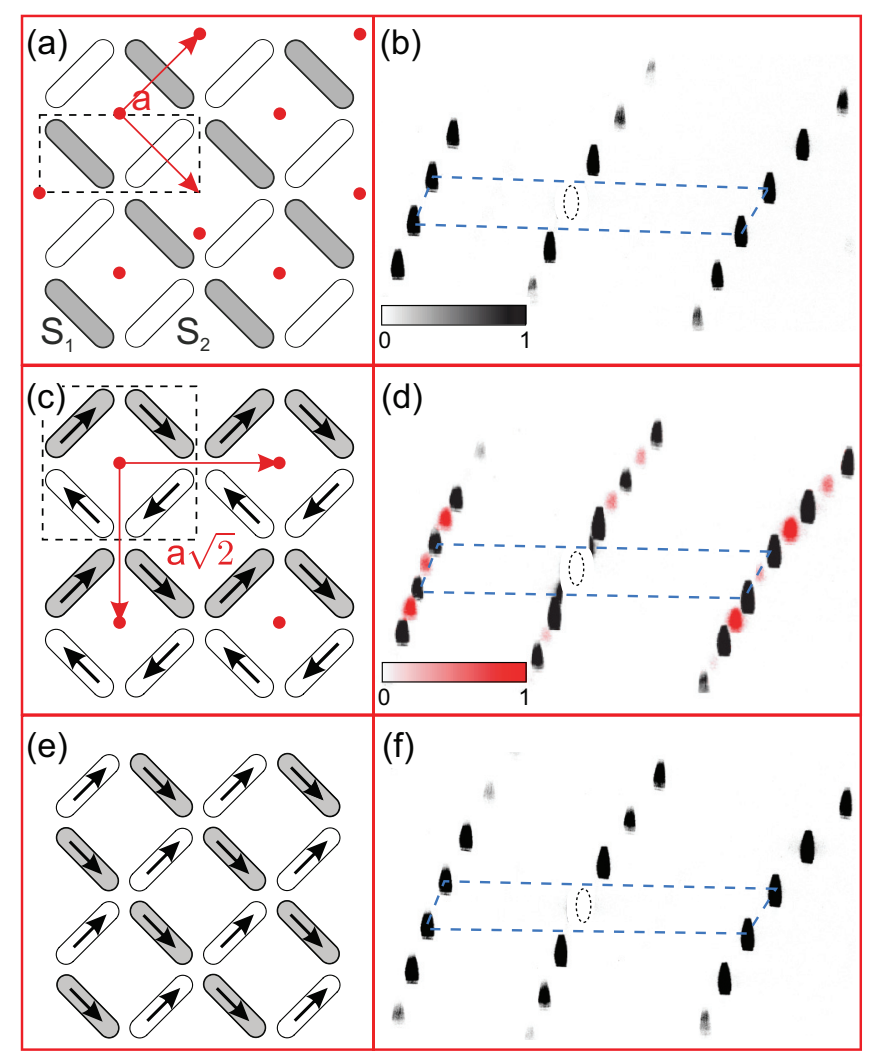

FIG. 2. (Color online) Scattering from as-grown artificial square ice. (a) Schematic of the sample geometry. The dashed box indicates the structural unit cell, containing two nanomagnets with different orientations. The corresponding two sublattices $S_{1}$ and $S_{2}$ are highlighted in gray and white, respectively. The red arrows represent the lattice vectors and the red dots indicate the square lattice, which has periodicity $a$. (b) Experimental scattering pattern recorded off resonance $(690 \mathrm{eV})$, containing structural information only. The first order Bragg peaks are indicated with a dashed box. The position of the specular reflection, which has been suppressed using a beam stop, is indicated with a dashed ellipse. (c) Schematic of the magnetic ground state, consisting of alternating rows of nanomagnets (highlighted in gray and white) with magnetic moments pointing towards opposite directions. The magnetic unit cell is indicated with the dashed box. (d) Experimental scattering pattern recorded at the $\mathrm{Fe} L_{3}$ edge $(706.8 \mathrm{eV})$, including both structural and magnetic information. The Bragg peaks highlighted in red are of pure magnetic origin and are a signature of long range ground-state ordering of the nanomagnet moments. The dashed box indicates the same first order Bragg peaks as in (b). (e) Schematic of a state at remanence following saturation. (f) Scattering pattern recorded at the $\mathrm{Fe} L_{3}$ edge $(706.8 \mathrm{eV})$ at remanence (zero field) after applying a magnetic field of $0.1 \mathrm{~T}$.

equal length $a \sqrt{2}$ and rotated by $45^{\circ}$ with respect to the ones of the structural lattice. The magnetic Bragg peaks thus have a periodicity of $2 \pi /(a \sqrt{2})$ and are placed exactly halfway between two structural ones.

\section{B. Magnetization reversal}

We apply a magnetic field along the diagonal of the square ice ( $y$ axis in our setup; see Fig. 1), i.e., at an angle of $\pm 45^{\circ}$ with respect to the long axis of the nanomagnets, 
which will cause the magnetic moments to rotate away from the nanomagnet easy axis. Therefore, we performed all scattering measurements at remanence in order to ensure that the moments are oriented parallel to the nanomagnet long axis, which facilitates interpretation of the data. Saturating the sample's magnetization along the $y$ axis $\left(H_{\mathrm{ext}}>800 \mathrm{Oe}\right)$ destroys the magnetic configuration of the as-grown state and, on the removal of the field, the magnetization direction of all nanomagnets remains oriented towards the direction of the previously applied external field as illustrated in Fig. 2(e). The magnetic and structural unit cells are now identical and the scattering pattern exhibits only Bragg peaks at the positions corresponding to the structural lattice as demonstrated by the on-resonance recorded pattern shown in Fig. 2(f).

In order to extract the magnetic contribution to each Bragg peak, we record scattering patterns with left and right circularly polarized light and then compute the dichroic contrast as defined in Eq. (1). The dichroic contrast characterizing the remanent state after magnetic saturation along the $y$ axis is shown in Fig. 3(a). Since all magnetic moments point towards the beam propagation direction, the observed dichroic contrast corresponds to its largest positive value, with all peaks in blue. Accordingly, the lowest negative value is observed after saturation along the negative $y$ direction, so that all peaks would appear red (not shown).

Applying a reverse magnetic field following saturation leads to changes in the dichroic scattering pattern, with particularly strong changes occurring for applied field values around the coercive field where the magnetization reversal takes place. As already observed in Ref. 24, the dichroic contrast does not change equally for all Bragg peaks, but can differ from peak to peak. It is via these differences that a detailed insight can be obtained in the reversal process. We demonstrate this in Fig. 3 by showing the hysteretic behavior of the dichroic intensity of four Bragg peaks [see Figs. 3(a), 3(c) and 3(d)]: the two first order peaks $P_{1}=(10)$ and $P_{2}=(01)$ and the two second order peaks $P_{3}=(1 \overline{1})$ and $P_{4}=(\overline{1} 1)$, respectively. The hysteresis curves of the first order peaks [Fig. 3(c)] reveal a small difference in their respective coercive fields of $H_{P_{1}}=475 \mathrm{Oe}$ and $H_{P_{2}}=455 \mathrm{Oe}$. The behavior of the two second order peaks $P_{3}$ and $P_{4}$ [see Fig. 3(d)] are identical within the experimental uncertainty with a coercive field value of $H_{P_{3}}=H_{P_{4}}=468 \mathrm{Oe}$ close to the average of the coercive fields of two first order peaks.

The observation of different coercive fields for the first order peaks $\left(P_{1}\right.$ and $\left.P_{2}\right)$ can be understood by assuming a slight misalignment between the sample axis and the direction of the applied magnetic field with $\phi_{1}>\phi_{2}$ as illustrated in Fig. 3(b). Such a misalignment will increase the field effectively applied along the long axis of the nanomagnets for the sublattice $S_{2}$ (in white), while for the case of the sublattice $S_{1}$ (in gray) it is decreased. Consequently, the magnetic moments on sublattice $S_{2}$ will tend to switch at lower external field values than the moments on $S_{1}$, so giving the small difference in the apparent coercive field. From the coercive field values we can estimate the angle of misalignment, which is smaller than $1.5^{\circ}$. The observation of different coercive fields for the two sublattices, as determined from the first order Bragg peaks $\left(P_{1}\right.$ and $P_{2}$ ), is in line with the absence of such a difference in (a)

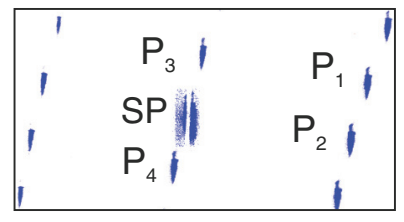

(b)
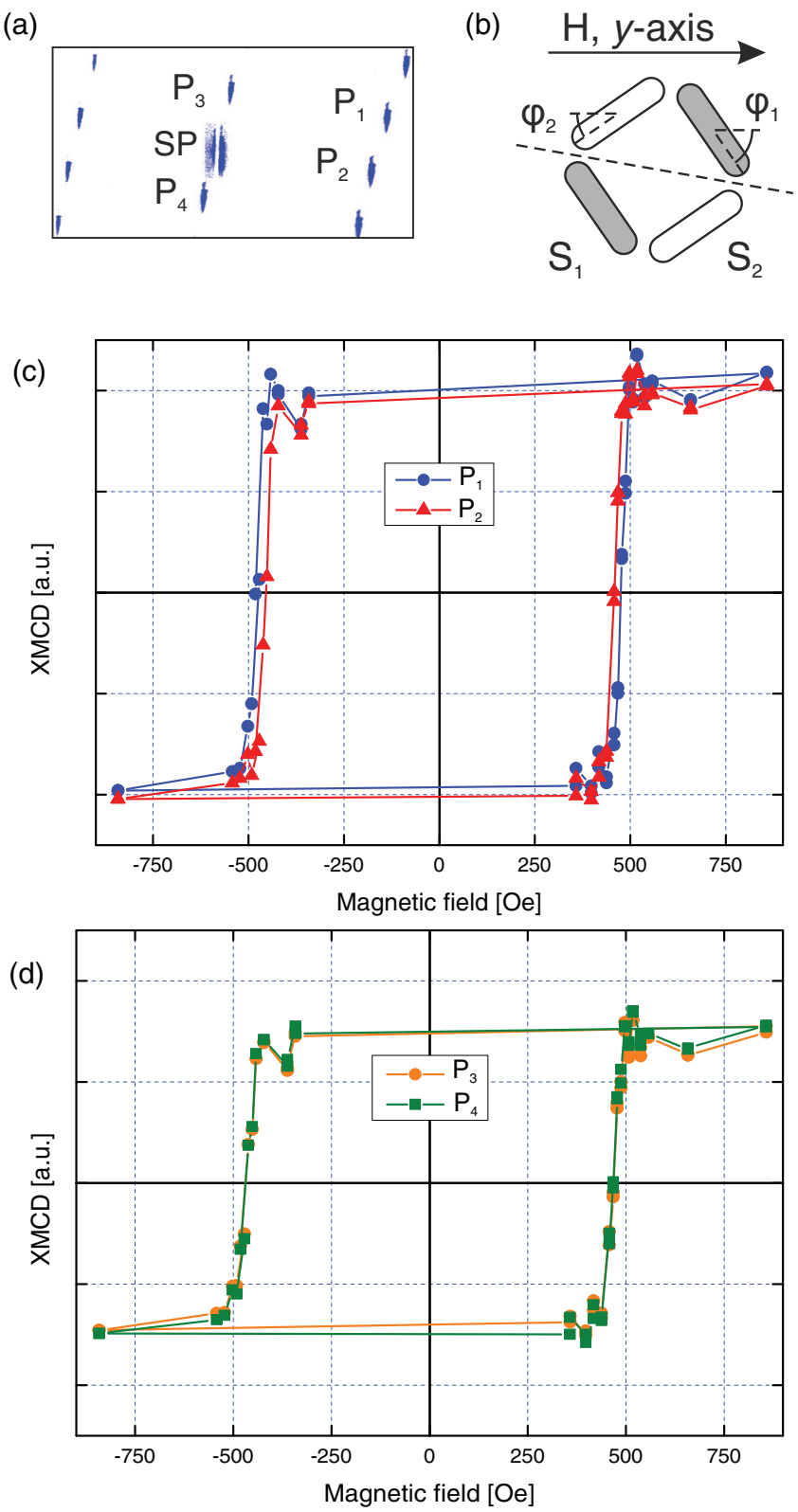

FIG. 3. (Color online) XMCD contrast at different Bragg peaks at remanence as a function of applied field. (a) Scattering pattern with the peaks considered, labeled $P_{1}$ to $P_{4}$ ( $S P$ indicates the specular reflection). (b) Schematic of the sample misalignment with respect to the applied field direction. The angle between the field direction and the nanomagnet long axis is bigger in the sublattice $S_{1}$ (indicated with $\varphi_{1}$ ) than in $S_{2}$ (indicated with $\varphi_{2}$ ). (c) XMCD contrast at the first order peaks $P_{1}$ and $P_{2}$. The two loops have different coercive fields, $H_{P_{1}}=475$ Oe and $H_{P_{2}}=455$ Oe, respectively. (d) XMCD contrast at the second order peaks $P_{3}$ and $P_{4}$. Here, the two curves are identical within the experimental error and the coercive field is $H_{P_{3}}=H_{P_{4}}=468 \mathrm{Oe}$.

the hysteresis curves of the two second order Bragg peaks $\left(P_{3}\right.$ and $P_{4}$ ), which sample both sublattices equally. Consequently, the field dependence of the dichroic contrast for $P_{3}$ and $P_{4}$ is directly proportional to the total magnetization of the array. 


\section{Determination of the nanomagnet contributions to the remanent magnetization}

The dichroic contrast of the Bragg peaks gives statistical information about the different configurations of the magnetic moments and, in this section, we show how one can derive separately the remanent magnetization $M_{r, 1}$ and $M_{r, 2}$ of the two sublattices $S_{1}$ and $S_{2}$ from the intensities of the Bragg peaks $P_{1}$ to $P_{4}$ shown in Fig. 3(a). The procedure consists of numerically computing the scattering patterns from test configurations of the nanomagnet moments, characterized by values of $M_{r, 1}$ and $M_{r, 2}$, followed by a comparison of the simulations with the experimental data and varying $M_{r, 1}$ and $M_{r, 2}$ until the best agreement is reached. This first requires the determination of the form factor of the nanomagnets and, once this is known, the total remanent magnetization $M_{r}$ is computed from the second order scattering peaks. Finally, the contributions to $M_{r}$ from each sublattice are derived from the different dichroic intensities of the first order peaks.

In order to numerically simulate the SXRMS pattern of our square ice arrays, the starting point is Eq. (2), which we evaluate following the approach presented in Ref. 28. While this approach was originally developed for scattering in the plane of incidence, we have adapted it to the present case of scattering in the full $q_{x}, q_{z}$ space. Since we are interested in the scattering from nanomagnets rather than from individual atoms, we replace in Eq. (2) the atomic form factor $f_{j}(E)$ with the form factor of the nanomagnets $F_{n}(\mathbf{q})$, which accounts for the scattering response of the entire nanomagnet. By summing over the nanomagnets, we obtain for the scattering intensity

$$
I(E, \mathbf{q}) \propto\left|\sum_{n} F_{n}(E, \mathbf{q}) \exp \left(i \mathbf{q} \cdot \mathbf{r}_{n}\right)\right|^{2} .
$$

Here the index $n$ refers to the $n$th nanomagnet, with center position $\mathbf{r}_{n}$. Assuming the nanomagnets to be homogeneous Permalloy particles, the form factor is given by

$$
F_{n}(E, \mathbf{q})=f(E) \int_{V_{n}} e^{i \mathbf{q} \cdot \mathbf{r}} d^{3} r
$$

where $f(E)$ is the atomic form factor as defined in Eq. (3). For the Fe $L_{3}$ edge at $706.8 \mathrm{eV}$, we use the values $f_{c}=10.554+$ $i 59.388$ and $f_{m}=-10.895-i 12.361$, which we determined using the method of Sève, Tonnerre, and Raoux. ${ }^{29}$

To evaluate the integral in Eq. (5), we approximate the shape of the nanomagnets with a cylinder of height $\mathbf{h}$ and elliptical cross section with semiaxes $\mathbf{a}$ and $\mathbf{b}$ as sketched in the inset of Fig. 1(a). We can then write the set of form factors $F_{n}(\mathbf{q})$ as $^{30}$

$$
F_{n}(\mathbf{q})=2 j_{0}\left(\mathbf{q} \cdot \mathbf{h}_{\mathbf{n}} / 2\right) \frac{j_{1}\left(\xi_{n}\right)}{\xi_{n}}
$$

with

$$
\xi_{n}=\sqrt{\left(\mathbf{q} \cdot \mathbf{a}_{\mathbf{n}}\right)^{2}+\left(\mathbf{q} \cdot \mathbf{b}_{\mathbf{n}}\right)^{2}},
$$

where $j_{0}$ and $j_{1}$ are spherical Bessel functions. As indicated in Fig. 2(a), the structural unit cell contains two structurally identical nanomagnets, but with different orientations. Their respective form factors $F_{1}(\mathbf{q})$ and $F_{2}(\mathbf{q})$ are defined by the following sets of vectors describing their spatial orientation:

$$
\begin{aligned}
& \mathbf{a}_{1}=\frac{230 \mathrm{~nm}}{2}\left(\frac{1}{\sqrt{2}}, \frac{1}{\sqrt{2}}, 0\right), \\
& \mathbf{b}_{1}=\frac{80 \mathrm{~nm}}{2}\left(-\frac{1}{\sqrt{2}}, \frac{1}{\sqrt{2}}, 0\right), \\
& \mathbf{h}_{1}=1 \mathrm{~nm}(0,0,1), \\
& \mathbf{a}_{2}=\frac{230 \mathrm{~nm}}{2}\left(-\frac{1}{\sqrt{2}}, \frac{1}{\sqrt{2}}, 0\right), \\
& \mathbf{b}_{2}=\frac{80 \mathrm{~nm}}{2}\left(\frac{1}{\sqrt{2}}, \frac{1}{\sqrt{2}}, 0\right), \\
& \mathbf{h}_{2}=1 \mathrm{~nm}(0,0,1) .
\end{aligned}
$$

We have set the relevant thickness of the nanomagnets to $1 \mathrm{~nm}$ in order to take into account that, due to the low penetration depth of soft $\mathrm{x}$ rays at small grazing angles, the scattering signal originates within the context of the kinematic scattering approximation from the first few atomic surface layers only. We finally note that for simplicity we did not try to reproduce the curvature of the scattering patterns that arises from projection of the Ewald sphere on the plane of the detector, and the nonparallel alignment of sample and detector with respect to the scattering plane.

In order to compare experimental and numerical scattering patterns, the dichroic contrast measured (or simulated) at remanence after applying a specific field $H$ has been normalized to the one measured (or simulated) at remanence following saturation, i.e., for each Bragg peak we considered the normalized dichroic contrast $\mathcal{I}_{\text {dic }}(H) \equiv I_{\text {dic }}(H) / I_{\text {dic }}\left(H_{\text {sat }}\right)$, which varies between +1 and -1 . Since the dichroic contrast of the second order peaks (either $P_{3}$ or $P_{4}$ ) is proportional to the total remanent magnetization $M_{r}, \mathcal{I}_{\text {dic }}$ at these peaks directly gives $M_{r}$ normalized to the saturation value, $\mathcal{I}_{\text {dic }}=$ $M_{r} / M_{\text {sat }} \equiv \mathcal{M}_{r}$. We therefore determine $\mathcal{M}_{r}$ from the average of $\mathcal{I}_{\text {dic }}$ at peaks $P_{3}$ and $P_{4} . \mathcal{M}_{r}$ takes the value of $+1(-1)$ when all moments are oriented towards the positive (negative) $y$ direction.

During magnetization reversal, the total magnetization is related to the number of nanomagnets $N^{-}$that have reversed their magnetic moments on application of a reverse magnetic field following saturation, so that $\mathcal{M}_{r}=\left(N^{+}-N^{-}\right) / N=$ $1-2 N^{-} / N$, where $N^{+}$is the number of nanomagnets that have not reversed their magnetic moments and $N=N^{+}+N^{-}$ is the total number of nanomagnets. $N^{-}$(and $N^{+}$) can therefore be calculated from $\mathcal{M}_{r}$ and the normalized dichroic intensity at the first order peaks $\left(P_{1}\right.$ and $\left.P_{2}\right)$ is now determined by the distribution of $N^{-}$nanomagnets between the two sublattices $S_{1}$ and $S_{2}$, i.e., by the values of $N_{1}^{-}$and $N_{2}^{-}$, with $N^{-}=N_{1}^{-}+$ $N_{2}^{-}$. We therefore generate trial configurations varying $N_{1}^{-}$ and $N_{2}^{-}$, and numerically compute the corresponding dichroic scattering patterns, comparing them to the experimental data until the best agreement for the asymmetry of dichroic intensity at $P_{1}$ and $P_{2}$ is obtained.

The validity of our method has been tested at different applied fields along the hysteresis loop. The numerical simulations reproduce quantitatively the dichroic intensity of the first and second order Bragg peaks, and we were also 


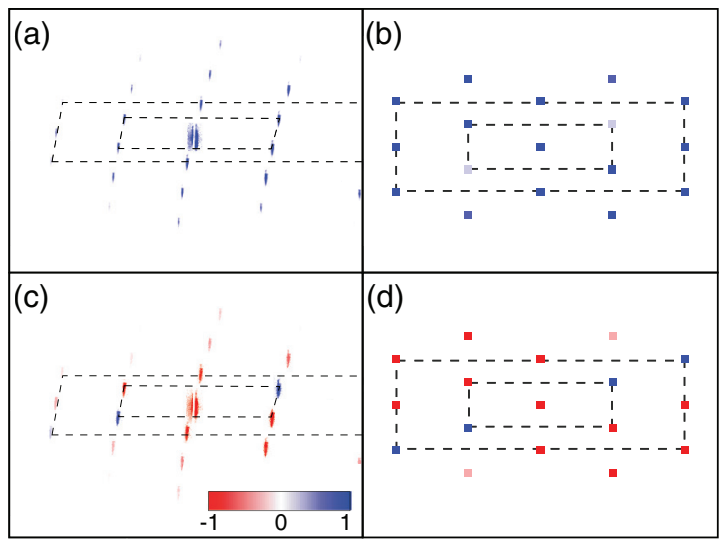

FIG. 4. (Color online) Comparison between experimental and numerical dichroic scattering patterns. Figures (a) and (c) have been measured at applied magnetic fields of 480 Oe and -472 Oe, respectively. The corresponding calculated best-fit patterns are shown in (b) and (d).

able to obtain qualitative agreement for the higher scattering order reflections. Two examples are shown in Fig. 4. Here we show two experimentally recorded dichroic patterns [panels (a) and (c)] with the best fits obtained by simulation [panels (b) and (d)]. In Fig. 4(a) the dichroic pattern was measured at remanence after application of a magnetic field of $H=+480$ Oe along the $+y$ direction (following saturation in the positive field direction). To obtain the most representative simulation shown in Fig. 4(b), $81 \%$ of the moments were reversed on sublattice $S_{1}$, and $72 \%$ on sublattice $S_{2}$. The corresponding numbers for the simulation shown in Fig. 4(d) are $73 \%$ and $20 \%$ for $S_{1}$ and $S_{2}$, respectively, which aim at reproducing the dichroic contrast shown in Fig. 4(c) recorded at remanence after applying an external field of $H=-472$ Oe (following saturation in the positive field direction).

When comparing the experiments and simulations of Fig. 4 in more detail, the differences in the dichroic intensities become more significant at the higher order peaks, even if the sign of the computed dichroic intensity is still correct for most of the observed peaks. This is not surprising given the simplicity of our model, and we can identify several causes. First, the higher scattering orders probe shorter length scales and are therefore more sensitive to the details of the shape of the nanomagnets. This includes not only deviations from the assumed cylindrical shape, but also small variations in size, shape, and microstructure between the nanomagnets themselves. Secondly, we note that our approach neglects the effects related to the refractive index, which give a modulation of the scattered intensity as a function of the direction of the reflected beam. This modulation is small close to the specular beam, but can be significant for higher order reflections. Nevertheless, for the determination of $M_{r, 1}$ and $M_{r, 2}$, a perfect agreement between simulations and experiments is not necessary, and the "qualitative" agreement at high scattering orders supports the validity of our model despite its simplicity.

\section{CONCLUSIONS}

We have investigated with soft $\mathrm{x}$-ray resonant magnetic scattering the magnetic configurations of an artificial square ice, both in its as-grown state and following the application of external magnetic fields. A two dimensional CCD detector allowed us to obtain in detail a large fraction of the sample's reciprocal space and unambiguously identify the positions of the Bragg peaks. The presence of long range magnetic ordering in the as grown sample was confirmed by the observation of pure magnetic scattering peaks only visible under resonance conditions, indicating a magnetic unit cell larger than the structural one. This magnetic ground state is lost after applying an external magnetic field. During magnetization reversal, we observed a different field dependence of the dichroic intensity in different first order Bragg peaks. We were able to reproduce this asymmetry with numerical simulations of the scattering patterns. This allowed us to determine separately the contribution to the total magnetization of the nanomagnets belonging to each one of the two sublattices of artificial square ice. Despite the simple approximations in the model underlying our simulations, we are able to reproduce the most important features of the magnetic dichroism in the experimental scattering patterns. This provides an important step forward, and will facilitate the interpretation of the scattering patterns of more complex spin ice systems such as the artificial kagome spin ice. ${ }^{7,31}$

We have demonstrated that significant insight into the magnetic configurations of artificial spin ice can be obtained by soft $\mathrm{x}$-ray resonant magnetic scattering, which opens the way to more sophisticated experiments such as coherent $\mathrm{X}$-ray scattering. X-ray photon correlation spectroscopy, for example, holds the promise of giving access to smaller length scales and shorter time scales than those accessible by realspace microscopy and will therefore play an important role in the further investigation of dynamics occurring in thermally activated artificial spin ice systems.

\section{ACKNOWLEDGMENTS}

The authors would like to thank A. Weber, V. Guzenko, and E. Deckardt for their help during sample preparation, V. Scagnoli and R. V. Chopdekar for useful discussions. We acknowledge Synchrotron SOLEIL for the provision of synchrotron radiation facilities and $\mathrm{H}$. Popescu for his support at the SEXTANTS beamline. B.T. acknowledges support by the ERASMUS Mundus India exchange program. The research leading to these results has received funding from the European Community's Seventh Framework Programme (FP7/2007-2013) under Grant No. 290605 (cofund: PSIFELLOW).

\footnotetext{
*luca.anghinolfi@psi.ch

${ }^{\dagger}$ Current address: Department of Physics, Jadavpur University, 700032 Kolkata, India.
}

${ }^{1}$ R. F. Wang, C. Nisoli, R. S. Freitas, J. Li, W. McConville, B. J. Cooley, M. S. Lund, N. Samarth, C. Leighton, V. H. Crespi, and P. Schiffer, Nature (London) 439, 303 (2006). 
${ }^{2}$ L. J. Heyderman and R. L. Stamps, J. Phys.: Condens. Matter 25, 363201 (2013).

${ }^{3}$ M. J. Harris, S. T. Bramwell, D. F. McMorrow, T. Zeiske, and K. W. Godfrey, Phys. Rev. Lett. 79, 2554 (1997).

${ }^{4}$ Y. Qi, T. Brintlinger, and J. Cumings, Phys. Rev. B 77, 094418 (2008).

${ }^{5}$ J. P. Morgan, A. Stein, S. Langridge, and C. H. Marrows, Nat. Phys. 7, 75 (2011)

${ }^{6}$ J. P. Morgan, J. Akerman, A. Stein, C. Phatak, R. M. L. Evans, S. Langridge, and C. H. Marrows, Phys. Rev. B 87, 024405 (2013).

${ }^{7}$ E. Mengotti, L. J. Heyderman, A. F. Rodriguez, F. Nolting, R. V. Huegli, and H.-B. Braun, Nat. Phys. 7, 68 (2011).

${ }^{8}$ S. D. Pollard, V. Volkov, and Y. Zhu, Phys. Rev. B 85, 180402(R) (2012).

${ }^{9}$ K. Zeissler, S. K. Walton, S. Ladak, D. E. Read, T. Tyliszczak, L. F. Cohen, and W. R. Branford, Sci. Rep. 3, 1252 (2013).

${ }^{10}$ U. B. Arnalds, A. Farhan, R. V. Chopdekar, V. Kapaklis, A. Balan, E. T. Papaioannou, M. Ahlberg, F. Nolting, L. J. Heyderman, and B. Hjorvarsson, Appl. Phys. Lett. 101, 4 (2012).

${ }^{11}$ A. Farhan, P. M. Derlet, A. Kleibert, A. Balan, R. V. Chopdekar, M. Wyss, L. Anghinolfi, F. Nolting, and L. J. Heyderman, Nat. Phys. 9, 375 (2013).

${ }^{12}$ J. M. Porro, A. Bedoya-Pinto, A. Berger, and P. Vavassori, New J. Phys. 15, 055012 (2013).

${ }^{13}$ M. Blume, J. Appl. Phys. 57, 3615 (1985).

${ }^{14}$ D. Gibbs, D. R. Harshman, E. D. Isaacs, D. B. McWhan, D. Mills, and C. Vettier, Phys. Rev. Lett. 61, 1241 (1988).

${ }^{15}$ J. P. Hannon, G. T. Trammell, M. Blume, and D. Gibbs, Phys. Rev. Lett. 61, 1245 (1988).

${ }^{16}$ J. P. Hannon, G. T. Trammell, M. Blume, and D. Gibbs, Phys. Rev. Lett. 62, 2644 (1989).

${ }^{17}$ B. T. Thole, G. van der Laan, and G. A. Sawatzky, Phys. Rev. Lett. 55, 2086 (1985).
${ }^{18}$ G. van der Laan, B. T. Thole, G. A. Sawatzky, J. B. Goedkoop, J. C. Fuggle, J.-M. Esteva, R. Karnatak, J. P. Remeika, and H. A. Dabkowska, Phys. Rev. B 34, 6529 (1986).

${ }^{19}$ G. Schütz, W. Wagner, W. Wilhelm, P. Kienle, R. Zeller, R. Frahm, and G. Materlik, Phys. Rev. Lett. 58, 737 (1987).

${ }^{20}$ J. M. Tonnerre, L. Sève, D. Raoux, G. Soullié, B. Rodmacq, and P. Wolfers, Phys. Rev. Lett. 75, 740 (1995).

${ }^{21}$ C. Spezzani, P. Torelli, R. Delaunay, C. F. Hague, F. Petroff, A. Scholl, E. M. Gullikson, and M. Sacchi, Physica B 345, 153 (2004).

${ }^{22}$ K. Chesnel, M. Belakhovsky, G. van der Laan, F. Livet, A. Marty, G. Beutier, S. P. Collins, and A. Haznar, Phys. Rev. B 70, 180402(R) (2004).

${ }^{23}$ C. Spezzani, M. Fabrizioli, P. Candeloro, E. Di Fabrizio, G. Panaccione, and M. Sacchi, Phys. Rev. B 69, 224412 (2004).

${ }^{24}$ J. P. Morgan, C. J. Kinane, T. R. Charlton, A. Stein, C. SánchezHanke, D. A. Arena, S. Langridge, and C. H. Marrows, AIP Adv. 2, 022163 (2012).

${ }^{25}$ N. Jaouen, J. M. Tonnerre, G. Kapoujian, P. Taunier, J. P. Roux, D. Raoux, and F. Sirotti, J. Synchrotron Radiat. 11, 353 (2004).

${ }^{26}$ M. Sacchi, N. Jaouen, H. Popescu, R. Gaudemer, J. M. Tonnerre, S. G. Chiuzbaian, C. F. Hague, A. Delmotte, J. M. Dubuisson, G. Cauchon, B. Lagarde, and F. Polack, J. Phys.: Conf. Ser. 425, 072018 (2013).

${ }^{27}$ L. Paolasini and F. de Bergevin, C. R. Phys. 9, 550 (2008).

${ }^{28}$ G. van der Laan, C. R. Phys. 9, 570 (2008).

${ }^{29}$ L. Sève, J. M. Tonnerre, and D. Raoux, J. Appl. Crystallogr. 31, 700 (1998).

${ }^{30}$ J. S. Pedersen, Adv. Colloid. Interfac. 70, 171 (1997).

${ }^{31}$ E. Mengotti, L. J. Heyderman, A. Fraile Rodríguez, A. Bisig, L. Le Guyader, F. Nolting, and H. B. Braun, Phys. Rev. B 78, 144402 (2008). 